\title{
Increased Facet Fluid Predicts Dynamic Changes in the Dural Sac Size on Axial-Loaded MRI in Patients with Lumbar Spinal Canal Stenosis
}

\author{
(D) Kanno, DH. Ozawa, DY. Koizumi, DN. Morozumi, DT. Aizawa, and DE. Itoi
}

\begin{abstract}
BACKGROUND AND PURPOSE: Axial-loaded MR imaging, which simulates the spinal canal in a standing position, demonstrates reductions of the dural sac cross-sectional area in patients with lumbar spinal canal stenosis. However, there has been no useful conventional MR imaging finding for predicting a reduction in the dural sac cross-sectional area on axial-loaded MR imaging. Previous studies have shown that increased facet fluid is associated with the spinal instability detected during positional changes. The purpose of this study was to analyze the correlations between facet fluid and dynamic changes in the dural sac cross-sectional area on axial-loaded MR imaging.
\end{abstract}

MATERIALS AND METHODS: In 93 patients with lumbar spinal canal stenosis, the dural sac cross-sectional area was measured by using axial images of conventional and axial-loaded MR imaging. Changes in the dural sac cross-sectional area induced by axial loading were calculated. The correlation between the facet fluid width measured on conventional MR imaging and the change in dural sac crosssectional area was analyzed. The change in the dural sac cross-sectional area was compared between the intervertebral levels with and without the facet fluid width that was over the cutoff value determined in this study.

RESULTS: The dural sac cross-sectional area was significantly smaller on axial-loaded MR imaging than on conventional MR imaging. The facet fluid width significantly correlated with the change in the dural sac cross-sectional area $(r=0.73, P<.001)$. The change in the dural sac cross-sectional area at the intervertebral level with the facet fluid width over the cutoff value was significantly greater than that at the other level.

CONCLUSIONS: The increased facet fluid on conventional MR imaging is highly predictive of the dynamic reduction of dural sac cross-sectional area detected on axial-loaded MR imaging in the clinical assessment of lumbar spinal canal stenosis.

ABBREVIATIONS: DCSA $=$ dural sac cross-sectional area; $L S C S=$ lumbar spinal canal stenosis; $R O C=$ receiver operating characteristic

M $\mathrm{R}$ imaging is widely used for the clinical assessment of degenerative lumbar spinal diseases. In evaluating the severity of spinal canal narrowing, the dural sac cross-sectional area (DCSA) is frequently measured by using axial MR images. ${ }^{1-6}$ However, conventional MR imaging is performed with the patient in the supine position, and the DCSA may be larger in this position than in the standing position. ${ }^{3,4,7}$ Hence, conventional

Received June 16, 2015; accepted after revision August 18.

From the Department of Orthopedic Surgery (H.K., H.O., T.A., E.I.), Tohoku University School of Medicine, Sendai, Japan; and Department of Orthopedic Surgery (Y.K., N.M.), Sendai Nishitaga National Hospital, Sendai, Japan.

This work is supported by a research grant from the Japan Orthopaedics and Traumatology Foundation, Inc (No. 205).

Paper previously presented at: Annual Meeting of the Spine Society of Europe, October 1-3, 2014; Lyon, France.

Please address correspondence to Haruo Kanno, MD, PhD, Department of Orthopaedic Surgery, Tohoku University School of Medicine, 1-1 Seiryo-machi, Aoba-ku, Sendai, 980-8574, Japan; e-mail: kanno-h@isis.ocn.ne.jp

http://dx.doi.org/10.3174/ajnr.A4582
MR imaging carries a risk of underestimating the severity of spinal canal narrowing. ${ }^{3,8}$

Recently, the clinical usefulness of axial-loaded MR imaging for assessing patients with lumbar spinal canal stenosis (LSCS) has been reported. ${ }^{3,4}$ With axial-loaded MR imaging, physiologically normal weight-bearing conditions in the upright position can be simulated by using a compression device with the patient in the supine position. Axial-loaded MR imaging may induce a significant reduction in the DCSA and potentially show additional imaging findings that cannot be acquired on conventional MR imaging. $^{3,4,6,8}$ The DCSA on axial-loaded MR imaging has been reported to correlate with the severity of clinical symptoms in patients with LSCS. ${ }^{9}$ Furthermore, previous studies have demonstrated that a dynamic decrease in the DCSA induced by axial loading increases the diagnostic specificity of spinal canal narrowing and influences the indications for surgical treatment. ${ }^{4,8,10,11}$ Therefore, evaluating the degree of spinal canal narrowing on axial-loaded MR imaging is beneficial for achieving a more accurate diagnosis and selecting the optimal treatment. However, no 

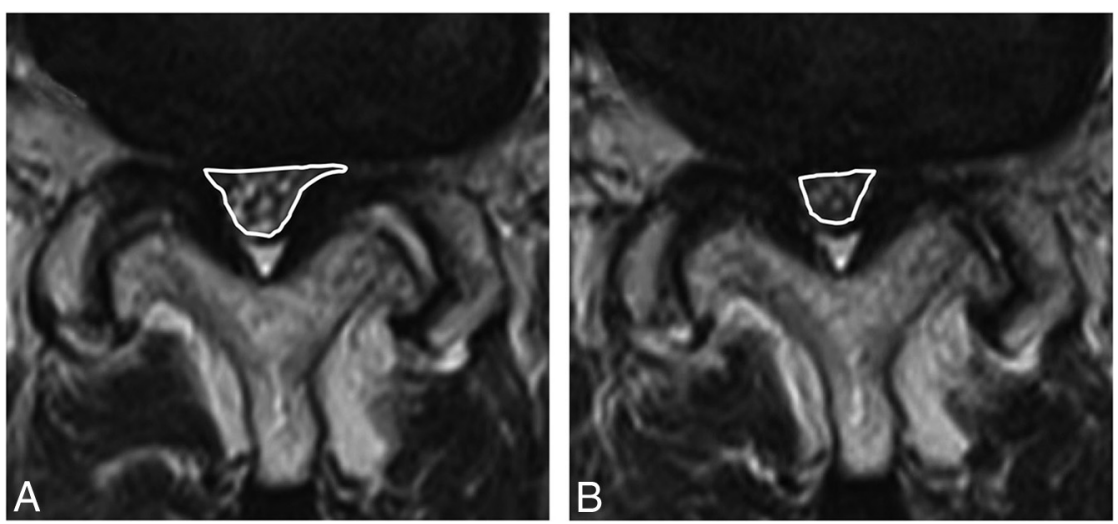

FIG 1. Measurement of the DCSA on conventional MR imaging and axial-loaded MR imaging in a representative case. The white lines indicate the outlines of the DCSA. The DCSA changed from $67(A)$ to $41 \mathrm{~mm}^{2}(B)$ due to axial loading.

reliable imaging findings on conventional MR imaging predict the dynamic reduction in the DCSA.

Many previous studies have shown that the morphology of the facet joints is associated with the segmental motion and instability of the lumbar spine detected during positional changes in the patient. ${ }^{12-15}$ Most interesting, recent studies have suggested that increased fluid signals in the facet joint on conventional MR images predict instability of the lumbar spine. ${ }^{16-19}$ Therefore, in the present study, we hypothesized that increased facet fluid signals on conventional MR images may be correlated with significant changes in the DCSA on axial-loaded MR images because the lumbar spinal canal is more likely to be affected by axial loading if the lumbar spine is unstable.

To the best of our knowledge, no previous studies have investigated the correlation between the facet fluid width and dynamic changes in the DCSA induced by axial loading. The purpose of this study was thus to analyze the correlation between the facet fluid width and dynamic changes in the DCSA detected by using axial-loaded MR imaging in patients with LSCS.

\section{MATERIALS AND METHODS}

\section{Patients}

This study prospectively included 93 consecutive patients (33 women and 60 men) referred to Sendai Nishitaga National Hospital for surgical treatment. The consecutive patients were enrolled in this study conducted between October 2007 and November 2009. All of the patients had neurogenic intermittent claudication and leg pain or numbness with associated neurologic signs ${ }^{20}$ in addition to radiographically confirmed lumbar spinal canal narrowing on cross-sectional imaging. In all cases, the neurologic diagnosis, such as lumbar radiculopathy and/or cauda equina syndrome, ${ }^{21,22}$ was confirmed by $>1$ spine surgeon on the basis of clinical symptoms (eg, intermittent claudication, leg pain/ numbness, and/or dysuria), neurologic findings (eg, muscle weakness, sensory disturbance, and/or abnormal tendon reflex), and imaging features, including assessments with plain radiography, CT, and MR imaging of the lumbar spine. The exclusion criteria were previous lumbar spine surgery, spondylolysis, disc herniation, severe osteoporosis, scoliosis, polyneuropathy, arterial insufficiency, and inflammatory/crystalline arthropathies.
Patients with congenital spinal anomalies and spinal deformities due to spinal trauma, infection, or tumor were also excluded. The institutional review board of Sendai Nishitaga National Hospital approved this study, and informed consent was obtained from all patients before participation. At the time of the initial evaluation, baseline characteristics, including age, sex, height, body weight, body mass index, and duration of symptoms, were registered for each patient.

\section{Conventional MR Imaging and Axial-Loaded MR Imaging}

MR imaging was performed by using a $1.5 \mathrm{~T}$ system (Magnetom Vision; Siemens, Erlangen, Germany) with a spine array coil. Axial T2weighted turbo spin-echo sequences were obtained. The TR and TE were 4000 and $120 \mathrm{~ms}$, the section thickness was $4 \mathrm{~mm}$, and the FOV was $200 \mathrm{~mm}$. The images were obtained at the middle of each intervertebral disc from L2-3 to L5-S1. ${ }^{7,9,23}$ The axial sections were placed as parallel to the intervertebral discs as possible by using sagittal T2-weighted imaging.

After the conventional MR imaging examination, axial loading was applied by using an external, commercially available nonmagnetic compression device, DynaWell L-Spine (DynaWell Diagnostics, Las Vegas, Nevada).,4 The compression force was approximately $50 \%$ of the patient's body weight, and loading was commenced 5 minutes before and continued during the MR imaging examination. Using a new sagittal T2-weighted image obtained in the axially loaded position, we again placed the axial sections in the plane of each disc. Both the axial-loaded and nonloaded MR images were acquired with straight knees to simulate a normal upright position. ${ }^{7,24}$ To prevent flexion of the spine during axial loading, we placed a cushion behind the lumbar spine. $^{7,25}$

\section{Measurements of the DCSA and Facet Fluid Width}

All measurements were obtained by using the OsiriX Imaging Software program, Version 2.7 (http:// www.osirix-viewer.com). The DCSA was measured from L2-3 to L5-S1 on the axial images (Fig 1). The measurements were acquired 3 times, and the mean value was calculated and used for the analysis. The measurements were performed by an experienced spine surgeon authorized by the Japanese Orthopaedic Association; the experimenter was blinded to which images were loaded or unloaded. The inter- and intraobserver reliability for the measurement of the DCSA was confirmed as excellent in our previous study. ${ }^{9,23}$ On the basis of the findings of previous studies, ${ }^{1,7,9,23}$ the DCSA at the most constricted intervertebral level was used to represent the degree of the spinal canal narrowing in each subject. The most constricted intervertebral level that presented with the smallest DCSA on conventional MR imaging was selected in each case for the data analysis.

According to previous reports, ${ }^{15,17}$ the facet fluid was defined as a high-intensity-signal area, closely matched to CSF on the axial 


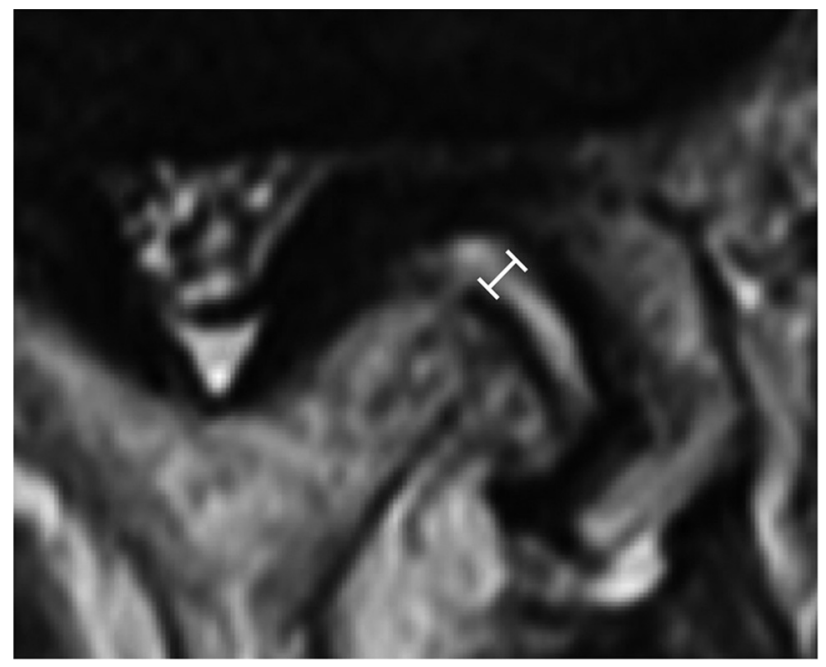

FIG 2. Measurement of the facet fluid width. The facet fluid width is defined as a high-intensity signal area, closely matched to the CSF on axial T2-weighted MR images. The measurements are recorded perpendicular to the joint line, and the largest value is documented as the facet fluid width (white line).

T2-weighted images obtained with conventional MR imaging. The facet fluid width was measured at the most constricted intervertebral level. The measurements were recorded perpendicular to the joint line, and the largest value was documented as the facet fluid width (Fig 2). ${ }^{15,17}$ The average of bilateral facet fluid width values was calculated for each patient and used for the data analysis.

This study also investigated the changes in the DCSA and the facet fluid width at normal or less severely stenotic intervertebral levels in the same subjects to compare with those at the narrowest level. One of the intervertebral levels excluding the narrowest level was randomly selected in each patient to prevent potential selection bias and increase comparability among the different intervertebral levels; then, the DCSA and the facet fluid width at the selected intervertebral level were determined as described above.

\section{Data Analysis}

The DCSA values were statistically compared between the conventional MR imaging and axial-loaded MR imaging. In addition, the changes in the DCSA between the conventional and axialloaded MR imaging were calculated. The Pearson correlation coefficient between the change in the DCSA and the facet fluid width was analyzed.

On the basis of the results of previous studies, a $>15 \mathrm{~mm}^{2}$ change in the DCSA induced by axial loading was defined as a significant change, ${ }^{3,4,8,11}$ worsening the severity of the clinical symptoms. ${ }^{9}$ The cutoff value for the facet fluid width providing the best combination of sensitivity and specificity for detecting a $>15 \mathrm{~mm}^{2}$ change in the DCSA at the most constricted intervertebral level was determined by using a receiver operating characteristic (ROC) analysis. The area under the ROC curve was also determined to evaluate the diagnostic power.

We confirmed the diagnostic capacity of the cutoff value for the facet fluid width determined according to the ROC analysis. All of the narrowest levels were divided into 2 groups: with facet fluid width over or under the cutoff value. Then, the DCSA on
Table 1: Baseline characteristics $(n=93)^{a}$

\section{Characteristic}

\section{Age (yr)}

Sex (male)

Height $(\mathrm{cm})$

Body weight $(\mathrm{kg})$

BMI $(\mathrm{kg} / \mathrm{m})$

Duration of symptoms (mo)

The most constricted level

L2-L3

L3-L4

L4-L5

L5-S1 $68 \pm 10$

$65 \%$

$160 \pm 9$

$64 \pm 11$

$25 \pm 3$

$32 \pm 33$

$1 \%$

$20 \%$

$71 \%$

$8 \%$

Note:-BMl indicates body mass index.

${ }^{a}$ Values are means or percentages.

conventional and axial-loaded MR imaging and the changes in the DCSA were compared between the groups.

To evaluate coexisting degenerative changes in the lumbar spine that may affect the facet fluid width and the spinal canal narrowing, we investigated the prevalence of a $\geq 3-\mathrm{mm}$ spondylolisthesis on $\mathrm{x}$-rays in the standing position ${ }^{23}$ and the degree of disc degeneration on the T2-weighted sagittal MR images ${ }^{26}$ and statistically compared them between the 2 groups.

\section{Statistical Methods}

All statistical analyses were performed by using GraphPad Prism software, Version 4.0c (GraphPad Software, San Diego, California). The differences in the DCSA were analyzed by using the unpaired $t$ test. Pearson correlation coefficients between the facet fluid width and the change in the DCSA were determined. The prevalence of the spondylolisthesis and the degree of disc degeneration were compared by using the $\chi^{2}$ test. A $P$ value $<.05$ was statistically significant.

\section{RESULTS}

\section{Baseline Characteristics and DCSA Values in All Patients}

The baseline characteristics and the most constricted intervertebral level in all patients are summarized in Table 1 . The most constricted level was more frequently observed at L3-L4 (20\%) and L4-L5 (71\%) levels compared with the L2-3 (1\%) and L5-S1 $(8 \%)$ levels. The DCSA on axial-loaded MR imaging (45 \pm 29 $\mathrm{mm}^{2}$ ) was significantly smaller than that observed on conventional MR imaging $\left(57 \pm 28 \mathrm{~mm}^{2}\right)$ at the most constricted level $(P<.05)$ (Fig 3A). At the other intervertebral levels excluding the most constricted level, the DCSA on axial-loaded MR imaging $\left(107 \pm 41 \mathrm{~mm}^{2}\right)$ was significantly smaller compared with that on conventional MR imaging $\left(101 \pm 40 \mathrm{~mm}^{2}\right)(P<.05)($ Fig $3 B)$. The change in the DCSA at the most constricted level $(13 \pm 23$ $\left.\mathrm{mm}^{2}\right)$ was greater than that at the other levels $\left(6 \pm 7 \mathrm{~mm}^{2}\right)$.

\section{Correlation between the Facet Fluid Width and the Changes in the DCSA}

The values for the facet fluid width and changes in the DCSA for each patient are shown in Fig 4. The narrowest intervertebral levels with a greater facet fluid width generally showed larger changes in the DCSA (Fig 4A). According to the Pearson correlation analysis, there was a significant and good correlation between the facet fluid width and the change in the DCSA $(r=0.73, P<.001)$ (Fig $4 A)$. In contrast, at the other intervertebral levels, there was a poor correla- 

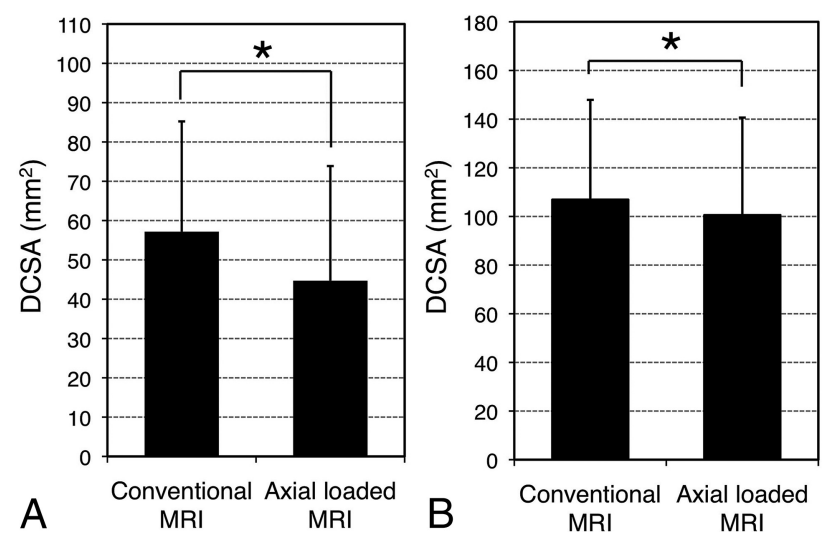

FIG 3. Comparison of the DCSA values between conventional and axial-loaded MR imaging. The DCSA on axial-loaded MR imaging is significantly smaller than that noted on conventional MR imaging at the narrowest intervertebral level $(A)$ and at the other level $(B)$. The asterisk indicates $P<.05$; the error bars, the SD.
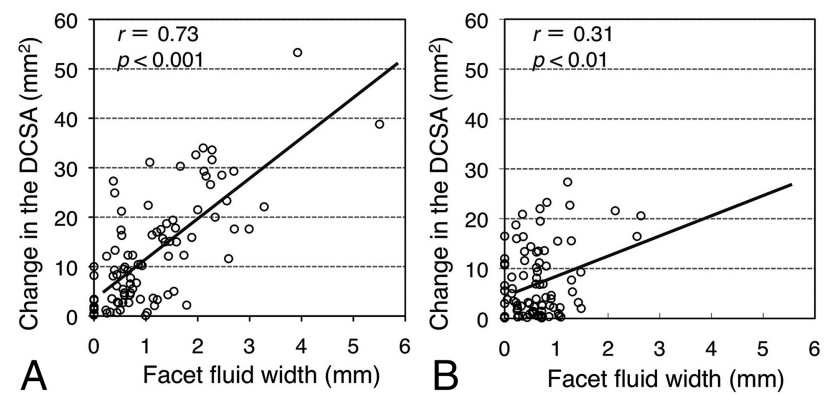

FIG 4. Facet fluid width and change in the DCSA in each patient. At the most constricted intervertebral level $(A)$, there is a significant and good correlation between the facet fluid width and the change in the DCSA (Pearson correlation coefficient $=0.73$ ). In contrast, there is a poor correlation at the other level $(B)$ (Pearson correlation coefficient $=0.31$ ).

tion between the facet fluid width and the change in the DCSA $(r=$ 0.31) (Fig 4B). The values of the change in the DCSA and the facet fluid width were generally smaller at the other intervertebral levels (Fig 4B) compared with the narrowest levels (Fig 4A).

\section{Diagnostic Power of the Facet Fluid Width for Detecting a $>15-\mathrm{mm}^{2}$ Change in the DCSA}

The ROC analysis showed that the facet fluid width at the most constricted intervertebral level could be used to detect a $>15 \mathrm{~mm}^{2}$ change in the DCSA, with $86.1 \%$ sensitivity and $84.2 \%$ specificity (Fig 5). The cutoff value for the facet fluid width was $1.04 \mathrm{~mm}$. The value of the area under the ROC curve (0.87) indicated that the facet fluid width was a valid discriminator of a $>15 \mathrm{~mm}^{2}$ change in the DCSA.

\section{Comparison of the Intervertebral Levels with the Facet Fluid Width over and under the Cutoff Value}

Among the most constricted intervertebral levels, facet fluid widths over and under the cutoff value of $1.04 \mathrm{~mm}$ were found at $41(44.1 \%)$ and $52(55.9 \%)$ levels, respectively. In the DCSA on conventional MR imaging, there was no significant difference between the levels with a facet fluid width over and under the cutoff value (Fig 6). On the other hand, the DCSA on axial-loaded MR imaging was significantly smaller at the levels with a facet fluid

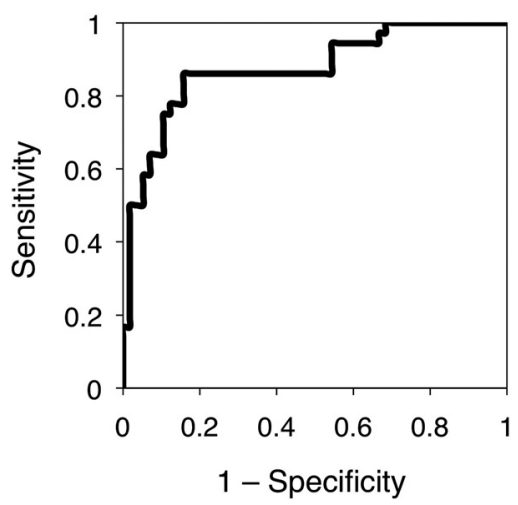

FIG 5. ROC curve for the facet fluid width for detecting a significant change in the DCSA at the most constricted intervertebral level. The area under the ROC curve is 0.87 .

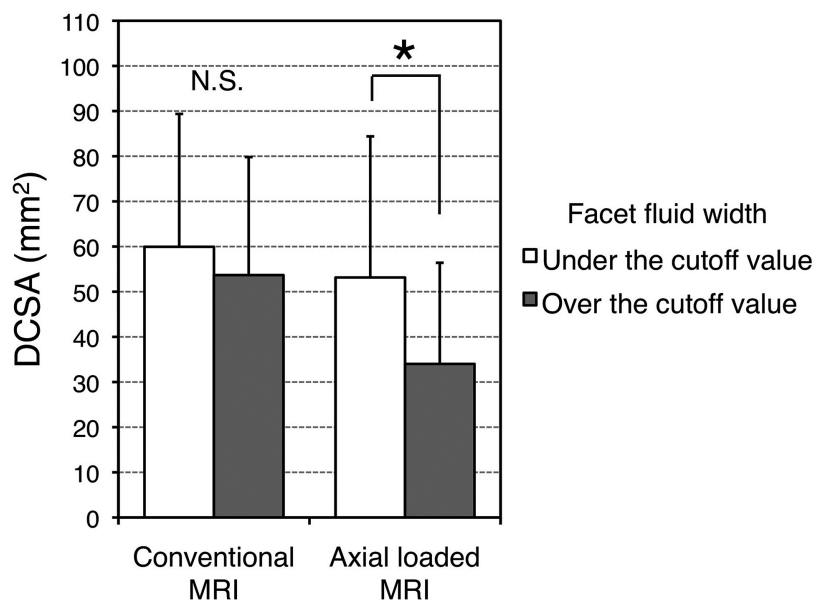

FIG 6. Comparison of the DCSA between the intervertebral levels with facet fluid widths over and under the cutoff value. In the DCSA on conventional MR imaging, there is no significant difference between the levels with facet fluid widths over and under the cutoff value of $1.04 \mathrm{~mm}$. On the other hand, the DCSA on axial-loaded MR imaging is significantly smaller at the levels with a facet fluid width over the cutoff value than those with a facet fluid width under the cutoff value. Asterisk indicates $P<.05$; error bars, the SD; N.S., not significant.

width over the cutoff value ( $\left.34 \pm 22 \mathrm{~mm}^{2}\right)$ than those with a facet fluid width under the cutoff value $\left(53 \pm 31 \mathrm{~mm}^{2}\right)(P<.01)$ (Fig $6)$. The levels with a facet fluid width over the cutoff value showed significantly larger changes in the DCSA $\left(20 \pm 11 \mathrm{~mm}^{2}\right)$ compared with those with a facet fluid width under the cutoff value $\left(7 \pm 6 \mathrm{~mm}^{2}\right)(P<.05)$ (Fig 7$)$.

The prevalence of spondylolisthesis was significantly higher among the intervertebral levels with a facet fluid width over the cutoff value $(73 \%)$ than among those with a facet fluid width under the cutoff value $(31 \%)(P<.001)$ (Table 2$)$. There was no significant difference in the degree of disc degeneration between the 2 groups (Table 3).

Among the other intervertebral levels excluding the narrowest level, facet fluid widths over and under the cutoff value were found at 15 (16\%) and 78 (84\%) levels, respectively. The change in the DCSA was significantly larger at the levels with a facet fluid width over the cutoff value $\left(11 \pm 9 \mathrm{~mm}^{2}\right)$ compared with those with a facet fluid width under the cutoff value $\left(5 \pm 7 \mathrm{~mm}^{2}\right)(P<$ $.01)$. 


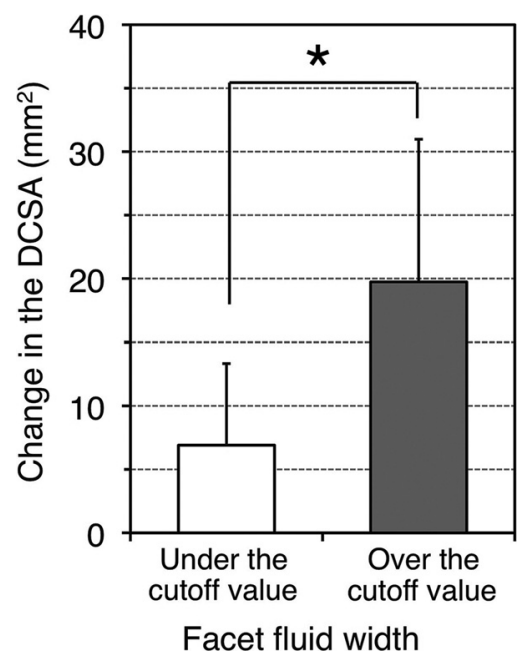

FIG 7. Comparison of the changes in the DCSA induced by axial loading between the intervertebral levels with facet fluid widths over and under the cutoff value. The levels with a facet fluid width over the cutoff value show significantly larger changes in the DCSA compared with those with a facet fluid width under the cutoff value. Asterisk indicates $P<.05$; error bars, the SD.

Table 2: Prevalence of spondylolisthesis at the intervertebral levels with facet fluid width over and under the cutoff value ${ }^{a}$

\begin{tabular}{lcc}
\hline & \multicolumn{2}{c}{ Facet Fluid Width } \\
\cline { 2 - 3 } & $\begin{array}{c}\text { Over the Cutoff } \\
\text { Value }(\boldsymbol{n}=\mathbf{4 1})\end{array}$ & $\begin{array}{c}\text { Under the Cutoff } \\
\text { Value }(\boldsymbol{n}=\mathbf{5 2})\end{array}$ \\
\hline With spondylolisthesis & $30(73 \%)$ & $16(31 \%)$ \\
Without spondylolisthesis & $11(27 \%)$ & $36(69 \%)$ \\
\hline
\end{tabular}

${ }^{a}$ Values are the number of intervertebral levels and percentages. There is a significant difference $\left(\chi^{2}\right.$ test, $\left.P<.001\right)$.

Table 3: Disc degeneration at the intervertebral levels with facet fluid width over and under the cutoff value ${ }^{a}$

\begin{tabular}{lcc}
\hline \multirow{2}{*}{$\begin{array}{c}\text { Classification of Pfirrmann } \\
\text { et a } \mathbf{2}^{26} \text { for Disc } \\
\text { Degeneration }\end{array}$} & \multicolumn{2}{c}{ Facet Fluid Width } \\
\cline { 2 - 3 } & $\begin{array}{c}\text { Over the Cutoff } \\
\text { Value }(\boldsymbol{n}=\mathbf{4 1})\end{array}$ & $\begin{array}{c}\text { Under the Cutoff } \\
\text { Value }(\boldsymbol{n}=\mathbf{5 2})\end{array}$ \\
\hline Grade 3 & $10(24 \%)$ & $9(17 \%)$ \\
Grade 4 & $22(54 \%)$ & $26(50 \%)$ \\
Grade 5 & $9(22 \%)$ & $17(33 \%)$ \\
\hline
\end{tabular}

${ }^{a}$ No intervertebral level was classified as grade 1 or 2 . Values are the number of intervertebral levels and percentages. There is no significant difference ( $\chi^{2}$ test, $P=$.46).

\section{DISCUSSION}

Previous studies have shown that axial-loaded MR imaging demonstrated a significant reduction in the size of the dural sac compared with conventional MR imaging. ${ }^{3,4}$ It has also been reported that axial-loaded MR imaging potentially produces additional imaging findings that cannot be obtained with conventional MR imaging. ${ }^{4,6,8,27}$ Most important, the dynamic change in the DCSA detected on axial-loaded MR imaging is more strongly correlated with the severity of clinical symptoms compared with that noted on conventional MR imaging in patients with LSCS. ${ }^{9}$ Furthermore, the use of axial-loaded MR imaging increases the diagnostic specificity for detecting degenerative diseases in the lumbar spine and influences the indications for surgical treatment. ${ }^{7,10}$ Therefore, evaluating the degree of narrowing of the lumbar spinal canal by using axial-loaded MR imaging may be beneficial for obtaining a more accurate diagnosis and selecting the optimal treatment. ${ }^{7,8,11,28}$ However, it remains unclear whether any specific pathologic factors increase the dynamic change in the DCSA induced by axial loading to the lumbar spine. It also remains unknown whether any useful imaging findings on conventionalloaded MR imaging predict the dynamic change in the DCSA.

Many previous studies have suggested that the presence of increased fluid signals in the facet joint on conventional MR images predicts instability of the lumbar spine. ${ }^{17-19}$ In the present study, we hypothesized that an increased facet fluid width may be correlated with the dynamic change in the DCSA on axial-loaded MR imaging because the morphology of the lumbar spinal canal is more likely to be affected by axial loading if the lumbar spine is unstable. Most interesting, our results showed that the facet fluid width strongly correlates with the change in the DCSA induced by axial loading at the most constricted intervertebral level. This finding indicates that patients with a greater facet fluid width tend to show larger changes in the DCSA. Thus, the increased facet fluid width associated with spinal instability may be, at least in part, a pathologic feature increasing the dynamic change in the dural sac size during axial loading in patients with LSCS.

In the current study, the cutoff value of $1.04 \mathrm{~mm}$ for the facet fluid width showed both high sensitivity $(86.1 \%)$ and specificity (84.2\%) for detecting a significant change in the DCSA. Indeed, this study demonstrated that the levels with a facet fluid width over the cutoff value showed significantly larger changes in the DCSA compared with the other levels. These results indicate that a cutoff value of $1.04 \mathrm{~mm}$ for the facet fluid width on conventional MR imaging is a useful finding for predicting a significant change in the DCSA detected by using axial-loaded MR imaging in the clinical assessment of patients with LSCS.

Previous studies have also suggested that axial-loaded MR imaging provides useful findings in making a more accurate diagnosis of lumbar degenerative diseases and can be helpful for determining the appropriate course of treatment. ${ }^{6-8,10,11,28}$ However, in the current study, similar to previous reports, ${ }^{4,7,9}$ not all patients showed a significant difference in the DCSA between conventional and axial-loaded MR imaging. Therefore, which patients actually require an examination with axial-loaded MR imaging in addition to conventional MR imaging should be clarified. The results of this study suggest that additional imaging examinations, such as those with axial-loaded MR imaging and upright myelography, should be considered in patients with a facet fluid width over the cutoff value on conventional MR imaging, to prevent the risk of underestimating the degree of spinal canal narrowing.

This study has several limitations. First, the study population included a small number of patients with LSCS, though all cases were consecutively included and analyzed prospectively. Baseline characteristics of the patient population such as height, weight, and body mass index will vary widely in different countries/nations; therefore, the results of this study may not be applicable to any other patient groups. This study did not evaluate an asymptomatic population for comparison. The lack of a control population limits the clinical implications and usefulness of the results in this study. Further study including an asymptomatic population will provide firm evidence to support the clinical significance of the results in the assessment of patients with suspected LSCS. 
Second, degeneration of the facet joint can be an important factor associated with the facet fluid width and segmental instability; therefore, it is potentially related to the change in the DCSA during axial loading. However, this study did not analyze facet joint degeneration. Third, configuration of the facet joint is different depending on various factors such as the intervertebral level and degree of joint degeneration. ${ }^{13}$ The method of measuring the facet fluid width may not be accurate in this study because the axial image is not perfectly perpendicular to the facet joint.

\section{CONCLUSIONS}

The present study demonstrated that the facet fluid width is significantly correlated with the change in the DCSA induced by axial loading. In this study, the patients with greater facet fluid widths tended to show larger changes in the DCSA. A cutoff value of $1.04 \mathrm{~mm}$ for the facet fluid width on conventional MR imaging may be a useful predictor of a significant change in the DCSA obtained on axial-loaded MR imaging in the clinical assessment of patients with LSCS. The present findings also suggest that additional imaging examinations, such as axial-loaded MR imaging and upright myelography, should be considered in patients with a facet fluid width over the cutoff value to prevent the risk of underestimating the degree of spinal canal narrowing and determine the optimal treatment.

Disclosures: Eiji Itoi-UNRELATED: Board Membership: Board of Trustees, J Shoulder Elbow Surg.

\section{REFERENCES}

1. Ogikubo O, Forsberg L, Hansson T. The relationship between the cross-sectional area of the cauda equina and the preoperative symptoms in central lumbar spinal stenosis. Spine 2007;32:142328; discussion 1429 CrossRef Medline

2. Hamanishi C, Matukura N, Fujita M, et al. Cross-sectional area of the stenotic lumbar dural tube measured from the transverse views of magnetic resonance imaging. J Spinal Disord 1994;7:388-93 Medline

3. Danielson BI, Willén J, Gaulitz A, et al. Axial loading of the spine during CT and MR in patients with suspected lumbar spinal stenosis. Acta Radiol 1998;39:604-11 CrossRef Medline

4. Willén J, Danielson B, Gaulitz A, et al. Dynamic effects on the lumbar spinal canal: axially loaded CT-myelography and MRI in patients with sciatica and/or neurogenic claudication. Spine 1997;22: 2968-76 CrossRef Medline

5. Sirvanci M, Bhatia M, Ganiyusufoglu KA, et al. Degenerative lumbar spinal stenosis: correlation with Oswestry Disability Index and MR imaging. Eur Spine J 2008;17:679-85 CrossRef Medline

6. Hansson T, Suzuki N, Hebelka H, et al. The narrowing of the lumbar spinal canal during loaded MRI: the effects of the disc and ligamentum flavum. Eur Spine J 2009;18:679-86 CrossRef Medline

7. Kanno $\mathrm{H}$, Endo $\mathrm{T}$, Ozawa $\mathrm{H}$, et al. Axial loading during magnetic resonance imaging in patients with lumbar spinal canal stenosis: does it reproduce the positional change of the dural sac detected by upright myelography? Spine (Phila Pa 1976) 2012;37:E985-92 CrossRef Medline

8. Willén J, Danielson B. The diagnostic effect from axial loading of the lumbar spine during computed tomography and magnetic resonance imaging in patients with degenerative disorders. Spine 2001; 26:2607-14 CrossRef Medline

9. Kanno H, Ozawa H, Koizumi Y, et al. Dynamic change of dural sac cross-sectional area in axial loaded magnetic resonance imaging correlates with the severity of clinical symptoms in patients with lumbar spinal canal stenosis. Spine (Phila Pa 1976) 2012;37:207-13 CrossRef Medline

10. Hiwatashi A, Danielson B, Moritani T, et al. Axial loading during MR imaging can influence treatment decision for symptomatic spinal stenosis. AJNR Am J Neuroradiol 2004;25:170-74 Medline

11. Willén J, Wessberg PJ, Danielsson B. Surgical results in hidden lumbar spinal stenosis detected by axial loaded computed tomography and magnetic resonance imaging: an outcome study. Spine 2008;33: E109-15 CrossRef Medline

12. Kong $\mathrm{MH}$, Morishita $\mathrm{Y}, \mathrm{He} \mathrm{W}$, et al. Lumbar segmental mobility according to the grade of the disc, the facet joint, the muscle, and the ligament pathology by using kinetic magnetic resonance imaging. Spine (Phila Pa 1976) 2009;34:2537-44 CrossRef Medline

13. Sato K, Wakamatsu E, Yoshizumi A, et al. The configuration of the laminas and facet joints in degenerative spondylolisthesis: a clinicoradiologic study. Spine (Phila Pa 1976) 1989;14:1265-71 CrossRef Medline

14. Fujiwara A, Lim TH, An HS, et al. The effect of disc degeneration and facet joint osteoarthritis on the segmental flexibility of the lumbar spine. Spine (Phila Pa 1976) 2000;25:3036-44 CrossRef Medline

15. Oishi $Y$, Murase M, Hayashi $Y$, et al. Smaller facet effusion in association with restabilization at the time of operation in Japanese patients with lumbar degenerative spondylolisthesis. J Neurosurg Spine 2010;12:88-95 CrossRef Medline

16. Cho BY, Murovic JA, Park J. Imaging correlation of the degree of degenerative L4-5 spondylolisthesis with the corresponding amount of facet fluid. J Neurosurg Spine 2009;11:614-19 CrossRef Medline

17. Chaput C, Padon D, Rush J, et al. The significance of increased fluid signal on magnetic resonance imaging in lumbar facets in relationship to degenerative spondylolisthesis. Spine (Phila Pa 1976) 2007; 32:1883-87 CrossRef Medline

18. Schinnerer KA, Katz LD, Grauer JN. MR findings of exaggerated fluid in facet joints predicts instability. J Spinal Disord Tech 2008;21: 468-72 CrossRef Medline

19. Rihn JA, Lee JY, Khan M, et al. Does lumbar facet fluid detected on magnetic resonance imaging correlate with radiographic instability in patients with degenerative lumbar disease? Spine (Phila $\mathrm{Pa}$ 1976) 2007;32:1555-60 CrossRef Medline

20. Verbiest $\mathrm{H}$. A radicular syndrome from developmental narrowing of the lumbar vertebral canal. J Bone Joint Surg Br 1954;36-B:230-37 Medline

21. Suzuki H, Endo K, Kobayashi H, et al. Total sagittal spinal alignment in patients with lumbar canal stenosis accompanied by intermittent claudication. Spine 2010;35:E344-46 CrossRef Medline

22. Sato K, Kikuchi S. Clinical analysis of two-level compression of the cauda equina and the nerve roots in lumbar spinal canal stenosis. Spine 1997;22:1898-903; discussion 1904 CrossRef Medline

23. Ozawa H, Kanno H, Koizumi Y, et al. Dynamic changes in the dural sac cross-sectional area on axial loaded MR imaging: is there a difference between degenerative spondylolisthesis and spinal stenosis? AJNR Am J Neuroradiol 2012;33:1191-97 CrossRef Medline

24. Lohman CM, Tallroth K, Kettunen JA, et al. Comparison of radiologic signs and clinical symptoms of spinal stenosis. Spine 2006;31: 1834-40 CrossRef Medline

25. Danielson B, Willén J. Axially loaded magnetic resonance image of the lumbar spine in asymptomatic individuals. Spine 2001;26: 2601-06 CrossRef Medline

26. Pfirrmann CW, Metzdorf A, Zanetti M, et al. Magnetic resonance classification of lumbar intervertebral disc degeneration. Spine (Phila Pa 1976) 2001;26:1873-78 CrossRef Medline

27. Saifuddin A, McSweeney E, Lehovsky J. Development of lumbar high intensity zone on axial loaded magnetic resonance imaging. Spine 2003;28:E449-51; discussion E451-52 CrossRef Medline

28. Saifuddin A, Blease S, MacSweeney E. Axial loaded MRI of the lumbar spine. Clin Radiol 2003;58:661-71 CrossRef Medline 